\title{
The behaviour of iron during giant impacts
}

\author{
ZHI LI $^{1}$, RAZVAN CARACAS ${ }^{1}$ AND FRANÇOIS \\ SOUBIRAN $^{2}$
}

\section{${ }^{1} \mathrm{CNRS}$}

${ }^{2} \mathrm{CEA}$

Presenting Author: zhi.li@ens-lyon.fr

The prevailing theory of the origin of the Moon is the giant impact hypothesis, in which a Mars-sized impactor collides with the proto-Earth in the late stage of accretion and the Moon is subsequently formed from the proto-lunar disk made of the ejected materials. As the laboratory-scale experiments are not able to simulate planetary-scale impacts, our understanding of the giant impact mostly comes from hydrodynamic simulations. However, the results of these simulations heavily depend upon the available equation of state to describe the thermodynamic response of the constituent materials of the proto-Earth and impactor to shock waves.

Iron as a building block material of the terrestrial planets naturally received significant attention. But the major effort has been put to determine its phase diagram up to the Earth's core conditions (126-360 GPa and 3000-7000 K) and beyond. The studies of iron at low densities are still scarce and the position of the critical point (CP) is uncertain. As the liquid-vapor dome ends at $\mathrm{CP}$, the position of the latter determines the time evolution of the proto-lunar disk during its condensation.

In order to assess whether the core of the planets undergoes significant vaporization during a giant impact, we employ $a b$ initio molecular dynamics and Monte Carlo simulations to explore iron over a wide density region encompassing the critical point (CP) and the Hugoniot lines of the shocked iron cores. Our results suggest that the iron core of the proto-Earth may become supercritical after giant impacts[1]. We also show that the iron core of Theia partially vaporized during the Giant Impact. Part of this vapour may have remained in the disk, to eventually participate in the Moon's small core. Similarly, during the late veneer stage a large fraction of the planetesimals have their cores undergoing partial vaporization. This would help to mix the highly siderophile elements into magma ponds or oceans.

Acknowledgements: This research was supported by the European Research Council (ERC) under the European Union's Horizon 2020 research and innovation programme (grant agreement $n^{\circ} 681818$ - IMPACT).

[1] Li, Caracas and Soubiran (2020), EPSL 547, 116463 